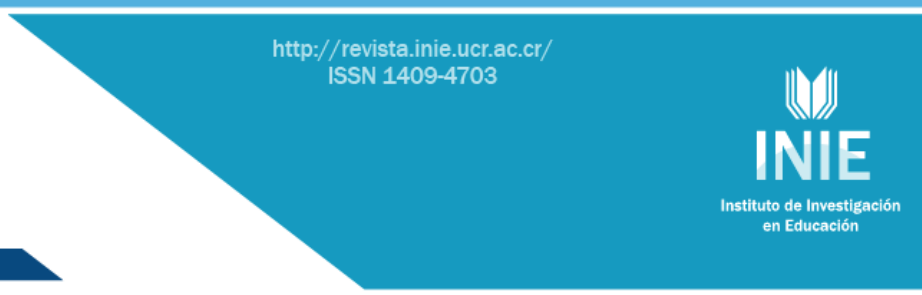

\title{
ESTILOS COGNITIVOS EN LA DIMENSIÓN CIENTÍFICA Y APORTACIONES A ESTILOS DE ENSEÑANZA EN DOCENTES UNIVERSITARIOS DE QUÍMICA
}

COGNITIVE STYLES IN SCIENTIFIC DIMENSION AND CONTRIBUTIONS TO THE

TEACHING STYLES OF UNIVERSITY CHEMISTRY PROFESSORS

\author{
Volumen 16, Número 2 \\ Mayo-Agosto
}

pp. 1-22

Este número se publicó el $1^{\circ}$ de mayo de 2016

DOI: http://dx.doi.org/10.15517/aie.v16i2.23995

Jaime Augusto Casas Mateus

Revista indizada en REDALYC, SCIELO

Revista distribuida en las bases de datos:

LATINDEX, DOAJ, E-REVIST@S, IRESIE, CLASE, DIALNET, SHERPA/ROMEO, QUALIS,

Revista registrada en los directorios:

ULRICH'S, REDIE, RINACE, OEI, MAESTROTECA, PREAL, CLACSO 


\title{
ESTILOS COGNITIVOS EN LA DIMENSIÓN CIENTÍFICA Y APORTACIONES A ESTILOS DE ENSEÑANZA EN DOCENTES UNIVERSITARIOS DE QUIIMICA \\ COGNITIVE STYLES IN SCIENTIFIC DIMENSION AND CONTRIBUTIONS TO THE TEACHING STYLES OF UNIVERSITY CHEMISTRY PROFESSORS
}

\author{
Jaime Augusto Casas Mateus ${ }^{1}$
}

\begin{abstract}
Resumen: Los estilos cognitivos y los de enseñanza han sido constructos objeto de investigación en las aulas universitarias. El presente ensayo pretende, primeramente, mostrar una condensada síntesis tanto de las tradiciones, desarrollos, enfoques y tendencias como de algunos estudios exploratorios, descriptivos o correlacionales relativos a los dos constructos, lo cual algunas veces implica el estudio de variables intervinientes. En segundo lugar, con una caracterización más integral y específica del profesorado universitario de química, este documento aborda dichos constructos; el estudio pretende ampliar el estado del arte, en dos vías: la primera toma en cuenta la dimensión científica en investigaciones anteriores a 1982 para el estudio del estilo cognitivo; la segunda tiene en cuenta la necesidad de la inclusión del ámbito del laboratorio para el estudio integral del estilo de enseñanza en este colectivo particular. Finalmente, el autor propone avanzar en el diseño y validación de instrumentos relativos a estos dos constructos $y$, posteriormente, estudiar las correlaciones entre el estilo de enseñanza, sea en el ámbito genérico (en la teoría) o en el ámbito específico (en el laboratorio) frente al respectivo estilo cognitivo en la dimensión científica en profesorado universitario de química.
\end{abstract}

Palabras clave: ESTILO COGNITIVO; ESTILO DE ENSEÑANZA; DIMENSIÓN CIENTÍFICA; DOCENCIA UNIVERSITARIA; QUIMICA.

\begin{abstract}
Cognitive and teaching styles have been construct objects of research in university classrooms. This article seeks first to show a condensed synthesis of traditions, developments, approaches and trends, as well as some exploratory, descriptive or correlational studies regarding these two constructs, which sometimes involves the study of intervening variables. Secondly, with a more integral and specific characterization of chemistry university professors, this document deals with those constructs; the study aims to broaden the state of the art in two ways: the first one takes into account the scientific dimension on research studies carried out before 1982 for the study of cognitive style; la segunda takes into account the need of including the laboratory field for the comprehensive study of the teaching style in this particular group. Finally, the author proposes advancing in the design and validation of instruments related to these two constructs and, after this step, studying the correlations between teaching style, whether in the generic field (in theory) or in the specific field (in the laboratory) regarding each cognitive style in the scientific dimension in university chemistry professors.
\end{abstract}

Key words: COGNITIVE STYLE; TEACHING STYLE; SCIENTIFIC DIMENSION; UNIVERSITY TEACHING; CHEMISTRY.

\footnotetext{
1 Profesor asociado del Departamento de Química de la Universidad Pedagógica Nacional, Colombia. Químico Farmacéutico de la Universidad Nacional de Colombia y Licenciado en Matemáticas de la Universidad Pedagógica Nacional en Bogotá, Colombia, Magister en Educación de la Pontificia Universidad Javeriana, Bogotá, Colombia y Doctorando en Educación de la Universidad Pedagógica Nacional.
}

Dirección electrónica: jcasas@pedagogica.edu.co

Ensayo recibido: 28 de junio, 2015

Enviado a corrección: 29 de febrero, 2016

Aprobado: 25 de abril, 2016 


\section{Introducción}

En el campo de la estilística son muchos los constructos que han emergido como definitorios de los patrones de conducta y de las particularidades de los sujetos en el ámbito educativo; este documento se centra en dos de ellos: el estilo cognitivo y el estilo de enseñanza. Estos dos conceptos han generado comprensiones e interpretaciones de la más diversa índole y actualmente se consideran tanto polisémicos como de gran relevancia en las connotaciones propias de sus dimensiones o categorías y respecto a las interrelaciones con otros dominios del ámbito escolar. En buena medida, la referencia a estos dos constructos tiene que ver con el interés de grupos de investigación por interpretar y correlacionar estos dos parámetros con factores de afectación de relaciones en el entorno educativo.

El acervo de investigaciones educativas en el campo de la estilística fue revisado por Hederich (2010) en la base de datos ERIC, Educational Resources Information Center. ERIC contiene el fichero de citas de documentos Resources in Education (RIE) y el fichero de citas de artículos de revistas Current Index to Journals in Education (CIJE), editado por el Departamento de Educación de Estados Unidos. Esta base de datos colecta 16 áreas temáticas distintas; se trata de la base de datos más importante en el mundo en el ámbito educativo, e incluye citas de artículos de revistas, informes técnicos, descripción de programas y materiales curriculares desde 1966 y que se actualiza trimestralmente.

Hederich (2010) encontró, haciendo un barrido desde 1991 hasta 2008, que la categoría que agrupa la mayor cantidad de publicaciones fue la de estilos cognitivos. Está seguida, de lejos, por la de estilos de enseñanza, la cual es la segunda en el examen comparativo de las publicaciones revisadas.

El presente ensayo es una aportación al estado del arte de estos dos marcos referenciales. Su perspectiva es proponer la inclusión de la 'dimensión científica' en el estudio del estilo cognitivo y, también, postular la inclusión del estilo de enseñanza en el escenario del laboratorio, hacia una definición más completa y coherente con la práctica de la enseñanza de la química en el profesorado universitario.

Para definir y estructurar el estado del arte se hizo la pesquisa bibliográfica respectiva en las siguientes bases de datos: EBSCOhost: Academic Source Complete, EBSCOHost: Education Research Complete, Jstor, E-libro, Ebrary, eBook collection, además del Portal de Revistas Académicas de la Universidad de Costa Rica, OPAC: Sistema de Bibliotecas Documentación e Información, y los Repositorios Institucionales del SIBDI (Kérwá). 
Adicionalmente se consultó en las bibliotecas estatales y universitarias colombianas que tienen acceso a centros de documentación en todo el mundo.

A continuación se muestra un recorrido por las comprensiones de los dos constructos a abordar: el de estilo cognitivo y, en menor medida, el estilo de enseñanza. Los dos constructos se estudian desde el ámbito educativo; se da cuenta de los desarrollos y tendencias desde sus inicios, pero resaltando la necesidad de ampliar los marcos de referencia hacia miradas más completas e inclusivas de las realidades de las prácticas de enseñanza universitarias, propias de disciplinas singulares como la química.

\section{Desarrollo del tema}

\subsection{Del estilo cognitivo}

\subsubsection{Tradiciones investigativas}

El estudio de los estilos cognitivos ha interesado históricamente a diversas ciencias, como la psicología o la antropología, siendo así que los estilos cognitivos hoy se entienden como estrechamente relacionados con la personalidad del individuo; en tal dirección, el constructo estilo cognitivo nace en el seno de la psicología cognitiva y se refiere a las distintas maneras en que las personas perciben la realidad de su entorno, procesan la información que se obtiene mediante la percepción, la almacenan en su memoria, la recuerdan y piensan sobre ella y se ha concebido como una dimensión sicológica que implica modos de funcionamiento cognitivo individual, relacionados con la adquisición y el procesamiento de información (Ausburn y Ausburn, 1978).

Messick (1976) -en concordancia con Witkin, Moore, Goodenough y Cox (1977)definió estilos cognitivos como actitudes, preferencias o estrategias habituales que determinan la manera como un sujeto percibe, recuerda, piensa y resuelve problemas.

Por otro lado, según Riding y Rayner (2002), el resultado de la suma del estilo cognitivo de una persona y su experiencia de aprendizaje da lugar a su estilo de aprendizaje, lo que en alguna medida explica la razón por la cual en el ámbito educativo, se ha estudiado más el estilo cognitivo en estudiantes que en docentes.

En el rastreo de las investigaciones sobre estilos cognitivos, se puede encontrar un importante inicio a finales de los años 40 y principios de los 50 , desde estudios que pretendían identificar diferencias individuales en la cognición con las características de ser estables, libres de valor, además de estar relacionadas con la personalidad y las relaciones sociales (Hanfmann, 1941; Klein, 1951; Klein y Schlesinger, 1951; Witkin, 1950; Witkin y Ash, 
1948). Tales estudios estaban enfocados en indagar respecto a etapas básicas o primarias de procesamiento de información, que incluían la percepción, la formación de conceptos, la clasificación y la categorización.

En referencia a las primeras investigaciones cabe citar a Hanfmann (1941), quien encontró que algunos sujetos empleaban una aproximación más perceptual que conceptual al ordenar objetos, al tratar de formular hipótesis sobre posibles agrupaciones. Witkin y Ash (1948) encontraron diferencias marcadas en la percepción de vertilcalidad en una prueba denominada test del marco y la varilla (rod and frame test) así como en otra prueba llamada test de ajuste corporal (body adjustment test).

En la primera prueba, se pedía colocar verticalmente una varilla giratoria que estaba en el centro de un marco luminoso inclinado; en la segunda, se pedía ajustar hasta la posición vertical la silla en la que el sujeto se encontraba sentado en una habitación con elementos inclinados. En estudios sobre estas pruebas y otras similares, las personas autoras encontraron que algunos sujetos privilegiaban guías visuales de paralelismo o perpendicularidad (presentes en el campo visual) para determinar la dirección de verticalidad (de la varilla inscrita en un marco rectangular, para el caso del test del marco y la varilla, por ejemplo), mientras que otros privilegiaban información de carácter vestibular, más relacionada con la dirección de la fuerza de gravedad.

En cualquiera de los casos presentados se evidenció que los sujetos privilegiaban información visual en un caso (llamados posteriormente dependientes del campo 'visual') o vestibular en el otro (llamados posteriormente independientes de campo); tales observaciones permitieron suponer que la percepción de verticalidad se podía indagar desde el estudio de tareas que implicaban la separación de un fragmento o desenmascaramiento de una figura del campo en el cual se encontraba inscrita, lo que se logró mediante el test de figuras enmascaradas o EFT (embedded figures test). Todos los estudios mencionados permitieron formular la dimensión perceptivo-analítica de una persona (en sujetos dependientes o independientes de campo) como una de las tipologías del estilo cognitivo (Witkin et al., 1954; citado en Hederich, 2004).

Es de anotar que el término estilo cognitivo en un principio no se había acuñado y, en este sentido, en los años 50 no existía una nominación unificada para tales diferencias individuales, por lo que se encuentran en la literatura nombres como actitudes perceptuales, patrones, predisposiciones, actitudes cognitivas, modos de respuesta o sistema de principios cognitivos (Holzman y Klein, 1954; Gardner, Holzman, Klein, Linton, y Spence, 1959). 
Además de lo anterior, cabe resaltar que a finales de los años 50, la noción de bipolaridad de Klein y Witkin (igualdad de valor a los polos de las dimensiones estilísticas) generó gran interés en la comunidad de investigadores sobre el estilo cognitivo. En esta dirección, y en concordancia con lo anterior, en los estudios de Wittkin et al. (1954) y de Klein y Schlesinger (1951) se evidenció una estrecha conexión entre inteligencia y personalidad, lo que generó la idea de que la 'disociación artificial' entre estos dos campos pudiera ser sorteada a partir de la noción de estilo cognitivo y, en tal dirección, los estudios insinuaban la posibilidad de describir a un sujeto como una entidad integral (Witkin et al., 1977).

A partir de este hecho, en la literatura aparece una serie de dimensiones o tipos de estilo cognitivo en la que se pretendía encontrar diferencias individuales en la cognición que fueran estables en el tiempo, libres de valor y ligadas a la personalidad, así como a las relaciones sociales. En consecuencia, es de entenderse que las tipologías de estilos cognitivos hayan sido enmarcadas desde la bipolaridad mencionada líneas atrás, en la que se engloba a la totalidad de los sujetos; así se pueden citar las dimensiones: DIC, dependencia-independencia de campo (Witkin, 1950), holístico-analítica (Riding, 2002), Reflexivo-impulsivo (Kagan, 1966) y otras.

Saliéndose de tal libreto, menos estudiados pero más propias del ámbito científico, surgen intentos por postular dimensiones asociadas con la esencia del conocimiento a incorporar, como las denominadas preferencias cognitivas, PC. Así, Witkin et al., (1977) afirmaron que las preferencias cognitivas eran un tipo de estilo cognitivo y representan lo que una persona típicamente hace con la información científica que recibe.

Conviene aquí explicar que el término 'preferencia cognitiva' fue introducido en un esfuerzo por evaluar los 'nuevos' currículos de ciencias, con la intencionalidad de enseñar al estudiantado cómo adquirir, evaluar y recuperar información, en contraste con la dinámica de memorización en currículos tradicionales propios de los años 60-70 en países como Estados Unidos, Reino Unido o Israel (Tamir, 1981). En tal sentido, Heath (1964), que fue quien las propuso, afirmaba que la interpretación cognitiva que cada estudiante exhibía cuando daba solución a un problema estaba ligada a la naturaleza del currículo; de conformidad con esta óptica, surgió una clasificación en 4 categorías: recordar información sin considerar sus implicaciones $(R)$, analizar la información con base en leyes o principios fundamentales $(P)$, relacionar la información con base en sus aplicaciones prácticas $(A)$ y efectuar cuestionamientos críticos con base en el contexto ( $Q$, por su sigla en inglés) (Heath, 1964). 
Es de notar que una gran cantidad de autores estudiaron las preferencias cognitivas, y sus avances se pueden resumir en la elaboración y validación de instrumentos (Tamir, 1981; McRobbie, 1991, Siegel y Siegel, 1965) para su medida y en la postulación de bipolaridades, tales como la R-Q y la P-A (Kempa y Dubé, 1973) así como en la comparación del constructo en varios grupos de química universitaria (Hofstein, Ben-Zvi y Samuel, 1978).

Cabe señalar que, con base en una revisión de la literatura efectuada respecto a preferencias cognitivas, dicho concepto fue dejado de estudiar desde el año 1982. Esto se debe en buena medida al menor interés sobre los currículos de ciencias, vistos ellos desde la óptica de las predilecciones del estudiantado de ciencias de accesar información científica a su haber cognitivo.

Otra de las aproximaciones al estilo cognitivo en lo tocante a la información científica es la propuesta del llamado 'Set Educacional', formulada por Siegel y Siegel (1965), quienes hipotetizaban que, mientras que las habilidades de una persona aprendiz serían un determinante del nivel de adquisición y comprensión de la información a ser aprendida, el 'Set Educacional' de cada estudiante determinaría la clase de material que prefería aprender y en dicha medida propusieron una estructura bipolar continua desde un aprendiz predispuesto a aprender información fáctica, hasta otro predispuesto a aprender información conceptual, en la que los sujetos (aprendices para este caso) se distribuían a lo largo de un continuo (Siegel y Siegel, 1965, citados en McRobbie, 1991).

En la Tabla 1 se presenta una síntesis de las categorías sobre estilos cognitivos en lo que se pudiera llamar la 'dimensión científica'. 
Tabla 1. Síntesis de categorías de estilos cognitivos en la 'dimensión científica' AUTOR DE CATEGORIA DESCRIPCIÓN REFERENCIA Heath (1964) Recordar, $R$ $<$ Recall, en la versión original, en inglés>

Aceptación de la información sin considerar sus implicaciones, aplicaciones o limitaciones (desde

Principios, $P$ $<$ Principles, en la versión original, en inglés>

Aplicación, A $<$ Application, en la versión original, en inglés>

Cuestionamiento, $Q$ $<$ Questioning, en la versión original, en inglés>

Siegel y Siegel

(1965) recordar dicha información).

Aceptación de la información en virtud a su ejemplificación o descripción de una relación, un concepto 0 un principio científico fundamental. Énfasis en la utilidad y aplicabilidad de la información en un contexto general, social o científico.

Cuestionamiento crítico de la
información, atendiendo a su exhaustividad, generalidad 0 limitaciones.

Predilección por recurrir a los hechos relacionados con la información científica.

Conceptual Predilección por recurrir a los conceptos relacionados con la información científica.

Fuente: elaboración propia a partir de Heath (1964) y Siegel y Siegel (1965).

En suma, se puede afirmar que estas primeras pesquisas dan cuenta de una investigación de corte básico sobre el estilo cognitivo y no muestran en ningún caso la intencionalidad de integración; además de lo anterior, se encuentran en el paradigma de enfrentar a un sujeto a dos o más maneras posibles de resolver una tarea, en situaciones de incertidumbre sobre la manera 'correcta' de resolverla. Dado que todos los modos de resolver la tarea se consideraban de igual valor, se asumía que en la prueba las personas revelaban una preferencia más no una habilidad; y quienes eran participantes quedaban divididos en dos grupos conformando los polos opuestos de un tipo particular de estilo.

Con base en lo anterior, se puede explicar el importante auge de instrumentos empleados en la determinación de una gran cantidad de estilos cognitivos (Witkin et al., 1954, para la dimensión dependencia/independencia de campo y Kagan (1966), para la dimensión reflexividad/impulsividad). En este período de relevante avance en el plano del diseño y estandarización de instrumentos, las investigadoras y los investigadores se encontraron con tres problemas (Kozhevnikov, 2007).

El primero tiene que ver con el hecho de que el principal enfoque en investigaciones sobre estilo cognitivo fuera el de dimensiones bipolares que representaban dos modos igualmente eficientes de resolver una tarea, pero que, en la realidad, mostraban que había 
uno de ellos más eficiente que otros. El segundo problema tenía que ver con el criterio de seleccionar la mediana como criterio de división para distinguir los grupos que representaban los polos opuestos de la dimensión (como para el caso de la dimensión reflexividad/impulsividad, Walker, 1976), que podía degenerar en que un sujeto perteneciera a un grupo en una investigación y en otra pudiera incluirse en la dimensión opuesta.

El tercer problema, que era el más importante en este período particular, tenía que ver con el hecho de encontrar una gran cantidad de estudios en la que los instrumentos se empleaban en investigaciones más de corte descriptivo y correlacional que explicativo, lo que da cuenta de una ausencia de investigación teórica en el campo que pudiera explicar la naturaleza subyacente al constructo o su relación con teorías de procesamiento de la información. Todo lo anterior es entendible en la medida que los instrumentos y los estudios fueron diseñados bajo el supuesto de que el estilo cognitivo estaba limitado a operaciones muy básicas de procesamiento de información (ver reviews de Kogan y Saami, 1990, y Sternberg y Grigorenko, 1997, citados en Kozhevnikov, 2007).

Retornando al barrido histórico de los estudios sobre estilos cognitivos cabe mencionar que en los años 70 se evidencia un descenso en la producción intelectual en lo que se pudiera denominar 'investigación básica' para dar paso a un número menor de investigaciones. Dichas pesquisas, presentadas por investigadores e investigadoras de campos aplicados, tales como la administración, la sicoterapia y la educación, estaban más bien enfocadas en relacionar el constructo con la ejecución de tareas más complejas (como la resolución de problemas, la toma de decisiones y el juzgamiento).

En virtud a la intencionalidad del presente escrito de abordar el estado del arte sobre estilos cognitivos centrándose en el ámbito educativo, solo se hace referencia tangencial a este último campo, que se constituye en el dominio más prolífico en investigaciones del estilo cognitivo. Esto se hace con el fin de comprender diferencias o preferencias individuales, pero enfocándose en los procesos de aprendizaje, lo que conllevó a la investigación sobre estilos de aprendizaje.

En esta dirección se pueden visualizar dos corrientes o aproximaciones para el estudio de los estilos de aprendizaje. Primero, se encuentra una de corte fenomenológico (Gregorc, 1985) en la que se postulan dos dimensiones: la percepción, que tiene que ver con la forma como los individuos capturan información, al catalogarla en concreta o abstracta; y el ordenamiento, que se relaciona con la manera en la que las personas organizan, sistematizan y emplean la información, que a su vez puede ser secuencial o aleatoria 
(Gregorc, 1985). La otra aproximación, formulada por Kolb (2005) consiste en un modelo de estilos de aprendizaje fundamentado en las teorías del aprendizaje experiencial, formuladas por John Dewey, Kurt Lewin y Jean Piaget (Kolb, Boyatzis y Mainemelis, 2001). Según Kozhevnikov (2007), las dos aproximaciones coinciden en presentar modelos de dos niveles de estilo cognitivo, uno de ellos en el plano de la percepción y otro en el plano de actividades cognitivas complejas, que involucran la toma de decisiones, el juzgamiento y la resolución de problemas.

En esta dirección, se puede encontrar otra línea de investigación centrada en la elaboración y estandarización de instrumentos para la medida de los estilos de aprendizaje. En esta línea se incluyen formatos como los cuestionarios de autoreporte, para poder abarcar las actividades cognitivas complejas mencionadas (Dunn, Dunn y Price, 1989; Lamb y Certo, 1978; Kolb, 2005; Kolb y Kolb, 2005).

El aporte de investigaciones aplicadas, como el de la pesquisa en educación que indaga por los estilos de aprendizaje, se encuentra matizado por la ausencia de distinción entre el constructo estilos de aprendizaje y la concepción básica de estilo cognitivo, que pudiera establecer una línea divisoria clara y así dar un marco teórico más sólido al estudio del constructo estilo cognitivo.

Kozhevnikov (2007) explica que desde los años 70 se evidencian tres tendencias en la investigación sobre estilos cognitivos, que se resumen en la Tabla 2. 
Tabla 2. Tendencias en la investigación de estilos cognitivos

Tendencia

\begin{tabular}{|c|c|c|}
\hline & & \\
\hline $\begin{array}{l}\text { Identificación } \\
\text { estilos que podían } \\
\text { operar en un nivel } \\
\text { metacognitivo }\end{array}$ & $\begin{array}{l}\text { Incluir, además del formato bipolar en algunas } \\
\text { dimensiones del estilo cognitivo, una estructura más } \\
\text { compleja que tiene en cuenta la rigidez/flexibilidad del } \\
\text { estilo, presentado por sujetos que en una situación se } \\
\text { podían incluir en el polo opuesto de una determinada } \\
\text { dimensión para relacionar el estilo cognitivo con una } \\
\text { función metacognitiva. }\end{array}$ & $\begin{array}{l}\text { Keller \& Ripoll, } \\
\text { 2001; Kholodnaya, } \\
\text { 2002; Niaz, 1987). }\end{array}$ \\
\hline $\begin{array}{lll}\text { Unificación } & \text { de } & \text { los } \\
\text { estudios en } & \text { una } \\
\text { estructura } & & \\
\text { multidimensional } & \end{array}$ & $\begin{array}{l}\text { Tendencia a sistematizar múltiples dimensiones } \\
\text { estilísticas en modelos coherentes y útiles en la } \\
\text { práctica, que emerge en los años } 90 \text {. Bajo la hipótesis } \\
\text { de que todos los estilos podían ser explicados por un } \\
\text { mismo fenómeno, con una variedad de elementos } \\
\text { subordinados basados en dimensiones estilísticas } \\
\text { existentes y algunos estilos nuevos. }\end{array}$ & $\begin{array}{l}\text { Allinson \& } \\
\text { Hayes, } \\
\text { Hermann, 1996; } \\
\text { Riding \& Cheema, } \\
\text { 1991, Sternberg \& } \\
\text { Grigorenko, 1997. }\end{array}$ \\
\hline $\begin{array}{l}\text { Interpretación de los } \\
\text { estilos cognitivos en } \\
\text { modelos jerárquicos } \\
\text { multinivel }\end{array}$ & $\begin{array}{l}\text { Sistematización de los estilos cognitivos en una } \\
\text { estructura compleja en el contexto de la teoría de la } \\
\text { información. Una de las propuestas (Miller, 1987, } \\
\text { 1991) se basa en la adición de una dimensión vertical, } \\
\text { con etapas de procesamiento cognitivo a la dimensión } \\
\text { holístico/analítica en la que cualquier tipología de estilo } \\
\text { cognitivo se podía ubicar en una celda de esta matriz. } \\
\text { Una segunda propuesta (Nosal, 1990) se soporta en la } \\
\text { tesis de que cualquier tipología del estilo cognitivo } \\
\text { podía ser interpretada como un elemento en una } \\
\text { matriz en que las filas constituyen niveles de } \\
\text { complejidad cognitiva y las columnas tipos de } \\
\text { procesos mentales. }\end{array}$ & $\begin{array}{l}\text { Miller (1987). Nosal } \\
\text { (1990) }\end{array}$ \\
\hline
\end{tabular}

Fuente: Elaboración propia a partir de Kozhevnikov (2007)

Kozhevnikov (2007) explica en detalle desarrollos en el campo de la Ciencia Cognitiva y en Neurociencia. Según la autora, dichos desarrollos intentan incorporar el estilo cognitivo dentro del dominio de la psicología cognitiva, pero no intentan desarrollar un marco teórico sólido a este constructo, aunque si dan luces para explicar el proceso neuronal subyacente a cada estilo cognitivo.

\subsubsection{Perspectivas de Investigación}

En el plano de las perspectivas investigativas, Kozhevnikov (2007) sugiere que el estilo cognitivo representa una heurística que un individuo emplea para procesar información acerca de su medio ambiente; también, menciona algunas vías de investigación promisorias en el estudio del estilo cognitivo, tales como el desarrollo de modelos matemáticos desde la sicometría, la neurociencia y la psicología social, que pueden aportar a la integración del 
constructo a las investigaciones sobre inteligencia, personalidad, ciencia cognitiva y educación y, además, ampliar los desarrollos de tales campos del conocimiento.

\subsection{Del estilo de enseñanza. Dos miradas: genérica y específica}

\subsubsection{La aproximación genérica}

Aún dentro del marco de la estilística se encuentra el constructo de estilo de enseñanza, que históricamente -como se mencionó en la introducción- ha constituido un tema de gran interés para la comunidad educativa. En términos generales, los estilos de enseñanza se han definido como un conjunto de orientaciones y actitudes que describen las preferencias de un sujeto cuando interactúa con un determinado contexto.

Casas y Guáqueta (2006) interpretan los estilos de enseñanza en términos de las particularidades y los patrones de conducta de los maestros en escenarios escolares. Abello y Hernández (2010), por otro lado, definen el estilo de enseñanza como un conjunto de acciones habituales que realiza cada docente en el aula, relativas a aspectos como la interacción con estudiantes, el grado de control y gestión del aula, y las estrategias de aula que este prioriza en el proceso de enseñanza-aprendizaje. Por su parte, Camargo y Hederich (2007) explican que las líneas de investigación en didáctica sobre estilos de enseñanza han estado enfocadas en dos miradas: la psicológica, en la que el estilo se define en términos de las formas preferidas de enseñar del docente que resultan connaturales al propio estilo cognitivo; y la pedagógica, en la que el estilo se enmarca en las necesidades de calificación del docente; además de lo anterior, los autores postulan una mirada comunicacional, que implica diferencias en las formas de interacción discursiva en el aula de clase y es aún incipiente, según las personas autoras.

Una vez definido el campo de trabajo y con base en la revisión bibliográfica efectuada, se evidencia una gran variedad de posturas y clasificaciones. Esto ha dado pie al establecimiento de un amplio número de categorías para la definición de tipologías sobre estilos y en algunos casos sobre métodos de enseñanza, como se muestra en la Tabla 3, en la que De León (2005) resume algunas de las aportaciones. 
Tabla 3. Resumen de la información reportada sobre Estilos de Enseñanza

\begin{tabular}{|c|c|c|c|}
\hline $\begin{array}{l}\text { AUTOR TIPO } \\
\text { DE ESTILO }\end{array}$ & $\begin{array}{l}\text { TIPO DE ESTILO Y } \\
\text { NIVEL }\end{array}$ & CLASIFICACIÓN & $\begin{array}{l}\text { CRITERIOS } 0 \\
\text { VARIABLES } \\
\text { CONSIDERADAS }\end{array}$ \\
\hline Bigge (1976) & $\begin{array}{c}\text { Estilo de enseñanza. } \\
\text { Nivel: no } \\
\text { especificado }\end{array}$ & $\begin{array}{l}\text {-Arquitecto cultural } \\
\text {-Líder democrático } \\
\text {-Conservador cultural } \\
\text {-Autocrático } \\
\text {-Democrático } \\
\text {-Tolerante }\end{array}$ & $\begin{array}{l}\text { Rol docente en la } \\
\text { transmisión cultural } \\
\text { Interacción docente- } \\
\text { estudiante }\end{array}$ \\
\hline Gage (1978) & $\begin{array}{c}\text { Estilo de enseñanza. } \\
\text { Nivel: no } \\
\text { especificado }\end{array}$ & No aplica & $\begin{array}{l}\text { Reestructuración cognitiva. } \\
\text { Provisión de modelos. } \\
\text { Condicionamiento. }\end{array}$ \\
\hline Gregorc (1985) & $\begin{array}{c}\text { Estilo de enseñanza. } \\
\text { Nivel: básica a } \\
\text { preuniversitaria }\end{array}$ & $\begin{array}{c}\text {-Concreto secuencial } \\
\text {-Abstracto secuencial } \\
\text {-Concreto casual } \\
\text {-Abstracto casual } \\
\end{array}$ & $\begin{array}{l}\text { Habilidad perceptiva. } \\
\text { Habilidad de organización } \\
\text { perceptiva. }\end{array}$ \\
\hline $\begin{array}{l}\text { Joyce y Weill } \\
\text { (1985) }\end{array}$ & $\begin{array}{l}\text { Métodos de } \\
\text { enseñanza. } \\
\text { Nivel: no } \\
\text { especificado }\end{array}$ & $\begin{array}{c}\text {-Procesamiento de la } \\
\text { información } \\
\text {-Personales } \\
\text {-Interacción social } \\
\text {-Conductistas }\end{array}$ & $\begin{array}{c}\text { Orientación del aprendizaje } \\
\text { (cognitiva y de interacción } \\
\text { grupal) }\end{array}$ \\
\hline Guerrero (1988) & $\begin{array}{l}\text { Estilo de enseñanza. } \\
\text { Nivel: superior }\end{array}$ & $\begin{array}{l}\text {-Instrumental } \\
\text {-Interactivo } \\
\text {-Individualista }\end{array}$ & $\begin{array}{c}\text { Concepción docente de la } \\
\text { educación. } \\
\text { Tipo de acción ejecutada } \\
\text { para lograr el fin. }\end{array}$ \\
\hline Coronado (1993) & $\begin{array}{l}\text { Estilo de enseñanza. } \\
\text { Nivel: superior }\end{array}$ & $\begin{array}{l}\text {-Comunicación pedagógica } \\
\text { abierta } \\
\text {-Comunicación pedagógica } \\
\text { autoritaria } \\
\end{array}$ & $\begin{array}{c}\text { Comunicación pedagógica } \\
\text { docente alumno. }\end{array}$ \\
\hline Grasha (1994) & $\begin{array}{c}\text { Estilo de enseñanza. } \\
\text { Nivel: pre } \\
\text { universitario }\end{array}$ & $\begin{array}{c}\text {-Experto } \\
\text {-Autoridad formal } \\
\text {-Modelo personal } \\
\text {-Facilitador } \\
\text {-Delegador }\end{array}$ & $\begin{array}{l}\text { Acción instruccional. } \\
\text { Interacción con el } \\
\text { alumnado. Administración } \\
\text { escolar. }\end{array}$ \\
\hline Guerrero (1996) & $\begin{array}{l}\text { Estilo de enseñanza. } \\
\text { Nivel: superior }\end{array}$ & $\begin{array}{l}\text {-Progresivo } \\
\text {-Academicista } \\
\text {-Tradicional }\end{array}$ & $\begin{array}{l}\text { Orientación del aprendizaje. } \\
\text { Rol de la persona docente. }\end{array}$ \\
\hline Brightman (2001) & $\begin{array}{l}\text { Estilo de enseñanza } \\
\text { aprendizaje. } \\
\text { Nivel: preuniversitario } \\
\text { superior }\end{array}$ & $\begin{array}{c}\text {-Introvertido } \\
\text {-Extrovertido } \\
\text {-Razonador } \\
\text {-Intuitivo } \\
\text {-Conceptual } \\
\text {-Sensitivo } \\
\text {-Evaluador } \\
\text {-Perceptivo } \\
\end{array}$ & $\begin{array}{l}\text { Presentación y } \\
\text { procesamiento de la } \\
\text { información. }\end{array}$ \\
\hline
\end{tabular}

Fuente: elaboración propia a partir de De León (2005).

Como se ve en esta tabla, se evidencia una aproximación genérica para el estudio del estilo de enseñanza. En esta aproximación existe un gran cúmulo de dimensiones y 
categorías, que incluyen otro tanto de autoras y autores que interpretan el estilo según una perspectiva particular o un rasgo en el comportamiento del profesor o la profesora.

Por su parte, Rendón (2013) afirma que las definiciones y aproximaciones tienen en cuenta aspectos tan particulares como la manera como cada docente asume la tarea educativa, como media los procesos de enseñanza-aprendizaje, como presenta la materia o como se relaciona con sus estudiantes, entre otros más de 20 descriptores. Para De león (2005) las tipologías se pueden inscribir en bloques de variables, que se pueden catalogar en uno de los siguientes puntos:

- Interacción docente-alumnado (comunicativa y procedimental)

- Orientación del aprendizaje, nivel de complejidad deseado y acciones instruccionales para lograrlo.

- Rol docente

- Orientación del aprendizaje y nivel de complejidad en el funcionamiento y organización perceptual deseado: relación con afectaciones ambientales, sociales y de interacción grupal.

- Concepción docente del fin educativo, así como de las acciones pertinentes.

- Rol del estudiantado

- Administración escolar

En resumen, se encuentra en la literatura gran cantidad de estudios sobre estilos de enseñanza en escenarios universitarios en los que se evalúa el constructo en su relación con el tipo de clase enseñada, la experiencia docente o la carrera desde donde se imparte en el que se da cuenta de investigaciones en varias latitudes, pero en cualquier caso los estudios son de corte exploratorio, descriptivo y en el mejor de los casos correlacional, como es el caso de Rendón (2010) y Borgobello, Peralta y Roselli (2010).

Con base en el objetivo del presente escrito, se resaltan dos posturas para investigar sistemáticamente el estilo de enseñanza. La primera de ellas es más general, en ella el concepto se describe en dimensiones que abarcan el ejercicio de la enseñanza en todas sus posibles manifestaciones; desde esta óptica cabe resaltar la propuesta de Abello, Hernández y Hederich $(2011,2012)$, que incluye tres dimensiones: social, control y estrategias de aula.

Con más detalle, Abello, Hernández y Hederich (2012), para la definición de categorías, postulan un modelo explicativo del constructo estilo de enseñanza desde la Teoría General de Sistemas de Bertalanfy (1976), quien interpreta el aula como un sistema. 
Las personas autoras de esta propuesta definen tres dimensiones que engloban las interacciones entre el docente, el estudiante y el grupo en su conjunto.

La primera de ellas, la dimensión social, es tipificada por dos subdimensiones: S1 o interacción docente-estudiante y S2 o toma de decisiones, extraídas de postulados de Grasha (2002), pero modificadas desde la comprensión del sistema aula.

La segunda es la dimensión control y gestión del aula, estructurada desde los postulados de Vásquez y Martínez (1996) que interpretan a la persona docente como quien tiene la autoridad y determina reglas; esta segunda dimensión se amplía desde las tesis de Blásquez, Fuentes y Costa (2004), quienes categorizan las reglas en explícitas/implícitas. Aparecen en esta dimensión dos subdimensiones: la C1 o estructuración de la enseñanza, que se refiere al nivel en el que el docente organiza o estructura los procesos de enseñanza, y la C2 o control del comportamiento, que se refiere al nivel de control sobre la acción del estudiante en clase.

La tercera es la dimensión estrategias de aula, que recoge dos bipolaridades activo/teórico (A/T) y reflexivo/pragmático (R/P), que a su vez surgen de la acción docente y en los comportamientos de enseñanza según el estilo de aprendizaje, soportadas en los planteamientos de Alonso, Gallego y Honey (1997), pero interpretadas para la enseñanza según Martínez (2009).

La segunda postura, más específica, incluye las particularidades de la enseñanza en el ámbito de laboratorio. Esta interpretación será explicada en la siguiente sección.

\subsubsection{La aproximación específica}

Además de la mirada genérica que recién se explicó, en la literatura se encuentra una mirada más específica, propia del dominio de conocimiento en el que se da la enseñanza. Bajo esta segunda postura, en estudios sobre estilos de enseñanza de las profesoras y los profesores en el laboratorio de ciencias, se pueden citar tres referentes que son mencionados por Flores, Caballero y Moreira (2009), quienes hicieron una revisión documental sobre la problemática de la enseñanza y el aprendizaje de las ciencias en el área de la química.

El primer autor referenciado es Domin (1999), quien categoriza los estilos en el laboratorio en expositivos, por descubrimiento, indagativos y por resolución de problemas. Para Domin (1999), el estilo denominado tradicional, expositivo o de verificación, sigue una rutina tipo "receta de cocina". Por su parte, el estilo por Indagación ilustra el tipo de estilo del 
profesorado que obliga a sus estudiantes a recolectar información necesaria para comprobar o refutar experimentalmente una tesis o un principio fundamental, en el que la enseñanza se basa en el supuesto de que el proceso de indagación imita la investigación científica, colocando al estudiantado en el rol de investigadoras e investigadores novel. Para Domin (1999), en el estilo por descubrimiento, los laboratorios se desarrollan sin ningún tipo de introducción teórica, esperando que con la experiencia y con discusiones post-laboratorio, el estudiantado pueda descubrir los fundamentos de los fenómenos de las leyes. Finalmente, el estilo por resolución de problemas está caracterizado por estar fundamentado en una situación problema que pueda ser desarrollada y resuelta desde una serie de lecturas y con ayuda del profesor, quien funge como guía y facilitador.

El segundo grupo de autores lo constituyen Moreira y Levandowski (1983), quienes definen estilos coherentes con tipos de laboratorio: programado (altamente estructurado), con énfasis en la estructura del experimento (se centra en el diseño de experimentos) o con enfoque epistemológico (se basa en la resolución de problemas).

El tercer autor es Kirschner (1992), quien reseña estilos correspondientes a tipos de laboratorio: formal o académico (laboratorio tradicional tipo "receta de cocina"), experimental (laboratorio abierto, inductivo, orientado al descubrimiento) y divergente (mezcla de los dos anteriores). En la Tabla 4 se presenta una síntesis de las tipologías del estilo de enseñanza en el marco del laboratorio.

Tabla 4. Resumen de la información reportada sobre estilos de enseñanza en el ámbito del laboratorio

\begin{tabular}{|c|c|l|c|}
$\begin{array}{c}\text { AUTOR TIPO } \\
\text { DE ESTILO }\end{array}$ & $\begin{array}{c}\text { ESTILO } \\
\text { INSTRUCCIONAL O } \\
\text { TIPO DE } \\
\text { LABORATORIO }\end{array}$ & \multicolumn{1}{c|}{ CLASIFICACIÓN } & \multicolumn{1}{c|}{$\begin{array}{c}\text { CRITERIOS O } \\
\text { VARIABLE(S) } \\
\text { CONSIDERADA (S) }\end{array}$} \\
\hline Domin (1999) & Estilo INSTRUCCIONAL & $\begin{array}{l}\text {-Expositivo } \\
\text {-Por descubrimiento } \\
\text {-Indagativo } \\
\text {-Por resolución de problemas }\end{array}$ & $\begin{array}{c}\text { Manera de estructurar } \\
\text { las prácticas de } \\
\text { laboratorio }\end{array}$ \\
\hline $\begin{array}{c}\text { Moreira y } \\
\text { Levandowsky } \\
(1983)\end{array}$ & Tipo de Laboratorio & $\begin{array}{l}\text {-Programado } \\
\text {-Con énfasis en la estructura } \\
\text { del experimento } \\
\text {-Con enfoque epistemológico }\end{array}$ & $\begin{array}{l}\text { Estructuración de los } \\
\text { experimentos y } \\
\text { empleo o no de la V } \\
\text { heurística de Gowin en } \\
\text { la resolución de } \\
\text { problemas. }\end{array}$ \\
\hline $\begin{array}{c}\text { Kirschner } \\
(1992)\end{array}$ & Tipo de Laboratorio & $\begin{array}{l}\text { Formal o académico } \\
\text { Experimental } \\
\text { Divergente }\end{array}$ & $\begin{array}{l}\text { Aproximación inductiva } \\
\text { o deductiva }\end{array}$ \\
\hline
\end{tabular}

Fuente: elaboración propia a partir de Flores, Caballero y Moreira (2009) 


\subsubsection{Perspectivas de Investigación}

En lo referente a las necesidades de profundización en el estudio del estilo de enseñanza, Hederich (2010) puntualiza sobre la inaplazable tarea de consolidar conocimiento en el campo, clarificando las dimensiones y definiendo para ellas sus características diferenciadoras, su estabilidad en el tiempo, su carácter neutral y su coexistencia con otras dimensiones. El profesor Hederich enfatiza en la problemática de los cuestionarios de autoreporte, la necesidad de incluir al estudiantado en la determinación del estilo de enseñanza de quien es docente y también en la utilidad y pertinencia de comparar los resultados obtenidos bajo la óptica de los dos actores del proceso educativo, con dos tipos de formato: uno para docentes y otro para estudiantes, en la mira de optimizar una más adecuada investigación en términos de la elaboración y estandarización de instrumentos para su medida; además de lo anterior, Hederich (2010) hace énfasis también en la necesidad de avanzar en los marcos explicativos de las relaciones entre estilo de enseñanza del docente y estilos cognitivos o de aprendizaje en sus estudiantes, para relacionarlos con su respectivo logro académico.

\section{Reflexiones finales. La propuesta}

El autor del presente escrito postula la necesidad de incluir la 'dimensión científica' en la tendencia a unificar el constructo del estilo cognitivo. Esta dimensión tendría en cuenta tanto funciones cognitivas básicas como el recordar o el fundamentarse en principios, así como funciones avanzadas, que implican la posibilidad de aplicar o cuestionar el conocimiento científico, para incorporar tal aproximación a sus desarrollos. Por otro lado, con base en las tradiciones evidenciadas en la literatura sobre el estudio del estilo de enseñanza para el ámbito de las ciencias naturales, el autor del presente documento postula también la necesidad de investigar el estilo de enseñanza en contextos propios de las disciplinas, como es el caso de la química, pero incluyendo el estilo en el ámbito de laboratorio, para centrar las investigaciones en el contexto de escenarios y realidades actuales, hacia una más adecuada caracterización del cuerpo docente universitario.

En la propuesta, tanto para el estilo cognitivo en la dimensión científica como para el estilo de enseñanza en el ámbito del laboratorio, se entiende la imperiosa necesidad de avanzar en el diseño, ajuste y validación de instrumentos para su medida, en colectivos tanto de docentes (para el estudio del estilo cognitivo) como para el profesorado y sus respectivos estudiantes (para el estudio del estilo de enseñanza en el laboratorio), lo que permitirá su 
empleo posterior en investigaciones que impliquen tales dominios, en el contexto de la disciplina de la química y en escenarios universitarios.

Finalmente, se postula la importancia de la investigación correlacional de corte exploratorio, en el que se han realizado algunos pocos estudios que muestran resultados promisorios que han mostrado correlaciones positivas y negativas, algunas de ellas estadísticamente significativas, entre categorías del estilo de enseñanza, tanto en la teoría como en el laboratorio y categorías del estilo cognitivo en la 'dimensión científica' (Angulo, Ariza y Casas, 2012; Casas, 2011; Casas y Cárdenas, 2011); el continuar con tales investigaciones dará cuenta de las lógicas que subyacen al proceder del profesorado universitario de química y de sus posibles efectos sobre sus estudiantes, en aras a efectuar avances para consolidar teoría dentro del marco de la estilística en el ámbito educativo.

La propuesta apunta en su conjunto a avanzar en una más completa caracterización del perfil docente y, en dicha medida, poder aportar a investigaciones posteriores en el plano de la explicación más que en el plano de la descripción, sobre resultados adversos en fenómenos como la deserción, la mortandad académica o la desmotivación para el aprendizaje de la química, entre otras. Todo lo anterior se propone con miras a redireccionar la enseñanza desde el perfeccionamiento docente y desde la unión de voluntades bajo la preocupación por el mejoramiento del proceso de aprendizaje en el estudiantado, en aulas universitarias del área de la química y en contextos reales del mundo de hoy.

\section{Referencias}

Abello, Diana, Hernández, Carolina y Hederich, Christian. (Junio, 2012) Características psicométricas del Instrumento de Estilos de Enseñanza de Abello, Hernández y Hederich. Ponencia presentada en el V Congreso Mundial de Estilos de Aprendizaje. 27-29 de junio, Santander, Colombia.

Abello, Diana, Hernández, Carolina y Hederich, Christian. (2011). Estilos de Enseñanza en docentes universitarios, propuesta y validación de un modelo teórico e instrumental. Revista Pedagogía y Saberes, (34), 141-154.

Abello, Diana y Hernández, Carolina. (2010). Diseño y Validación de un modelo Teórico e Instrumental para la identificación de Estilos de Enseñanza en docentes universitarios. (Trabajo de Maestría no publicado). Universidad Pedagógica Nacional, Bogotá, Colombia.

Alonso, Catalina, Gallego, Domingo y Honey, Peter. (1997). Los estilos de aprendizaje. Bilbao: Ediciones Mensajero. 
Allinson, John \& Hayes, Christopher. (1996). The Cognitive Style Index, a measure of intuition-analysis for organizational research. Journal of Management Studies, 33(1), 119-135.

Angulo, Diego, Ariza, Hugo y Casas, Jaime. (Junio, 2012). Estilos de enseñanza en el laboratorio, una mirada al campo de la enseñanza de la química a nivel experimental. Ponencia presentada en el IX taller internacional "ENFIQUI 2012" "la enseñanza de la física y la química" y II taller "la enseñanza de las ciencias naturales", Matanzas, Cuba.

Ausburn, Lynna J., \& Ausburn, Floyd. B. (1978). Cognitive styles: Some information and implications for instructional design. Educational Communication and Technology, 26(4), 337-354.

Bertalanfy, Ludwig. (1976). Teoría General de Sistemas. Petrópolis: Vozes.

Bigge, Morris. (1976). Teorías de Aprendizaje para Maestros. México: Editorial Trillas.

Blásquez, Alicia, Fuentes, María y Costa, Albert. (2004). Estudio de la adaptación de familias inmigradas utilizando indicadores basados en mitos, reglas y rituales. (Tesis de maestría). Universidad Autónoma de Barcelona, Barcelona, España.

Borgobello, Ana, Peralta, Nadia y Roselli, Nestor. (2010). El estilo docente universitario en relación al tipo de clase y a la disciplina enseñada. Liberabit, 16(1), 07-16.

Brightman, Harvey. (2001). GSU Master Teaching Program: On Learning Styles; Student Learning and the Miers-Briggs Type Indicator. Recuperado de http://www2.gsu.edu/ dschjb/wwwmbti.html

Camargo, Angela y Hederich, Christian. (2007). El estilo de enseñanza, un concepto en búsqueda de precisión. Pedagogía y saberes, (26), 31-40.

Casas Jaime y Cárdenas, Fidel. (2011). Preferencias cognitivas y estilos de enseñanza en acción. Una mirada a la enseñanza del laboratorio de química. Taller presentado en el Quinto Congreso Internacional sobre formación de profesores de ciencias. Bogotá.

Casas, Jaime y Guáqueta, Sandra. (2006). Aproximaciones didácticas al concepto de estilo de enseñanza en docentes universitarios. Tecné, epistemé y didaxis, (24), 22-37.

Casas, Jaime. (2011). Una aproximación al estudio de los estilos de enseñanza, los estilos cognitivos, (en la dimensión dependiente e independiente de campo, DIC) y sus relaciones en maestros de química: un estudio de caso. Ponencia presentada en el Seminario Internacional Química: Historia, Filosofía y Educación. Bogotá, Colombia.

Coronado, Humberto. (1993). Caracterización de los Estilos Pedagógicos predominantes en la enseñanza de las Ciencias Biológicas en el Noveno Grado de educación básica. (Trabajo de Maestría no publicado). Universidad Pedagógica Experimental Libertador, Instituto Pedagógico de Maturín, Caracas, Venezuela.

De León, Iván J. (2005). Los estilos de enseñanza pedagógicos: una propuesta de criterios para su determinación. Revista de Investigación, (57), 69-97. 
Domin, Daniel. (1999). A review of laboratory instruction styles. Journal of Chemical Education, 76(4), 543-547.

Dunn, Rita., Dunn, Kenneth., \& Price, Gary E. (1989). Learning Style Inventory. Lawrence, KS: Price Systems.

Flores, Julia, Caballero, María C. y Moreira, Marco A. (2009). El laboratorio en la enseñanza de las ciencias: una visión integral en este complejo ambiente de aprendizaje. Revista de Investigación, 33(68), 75-111.

Gage, Nathaniel. (1978). Aprendizaje Escolar y Evaluación. Buenos Aires: Editorial Paidós.

Gardner, Riley, Holzman, Philip, Klein, George, Linton, Harrie \& Spence, Donald. (1959). Cognitive control. A study of individual consistencies in cognitive behavior (Part 4. Psychological issues). New York: International Universities Press.

Grasha, Anthony. (1994). A Matter of Style: The Teacher as Expert, Formal Authority, Personal Model, Facilitator and Delegator. College Teaching, 42(4), 142-149.

Grasha, Anthony. (2002). Teaching with Style. San Bernardino: C.A. Alliance Publishers.

Gregorc, Anthony. (1985). Teaching's Style (Gregorc's Mediation Abilities). En Pat Burke Guild and Stephen Garger (Eds.), Marching to Different Drummers (Chapter 10). Virginia, USA: Association for Supervision and Curriculum Development.

Guerrero, Betty. (1988). Estilos de Enseñanza y Formación Profesional Docente en educación superior en Venezuela. El Estilo de Enseñanza de Docentes en Institutos y Colegios Universitarios. (Trabajo de Ascenso a docente titular). Colegio Universitario de los Teques, Venezuela.

Guerrero, Nidia. (1996). Evaluación de los Estilos de Enseñanza y los Estilos de Aprendizaje como vía para mejorar la calidad de la instrucción en el Colegio Universitario de Los teques Cecilio Acosta. (Trabajo de grado de Maestría no publicado). Universidad Pedagógica Experimental Libertador, Instituto Pedagógico de Miranda "José Manuel Siso Martínez", Caracas Venezuela.

Hanfmann, Eugenia. (1941). A study of personal patterns in an intellectual performance. Character and Personality, 9(4), 315-325.

Heath, Robert. (1964). Curriculum, Cognition and Educational Measurement. Educational and psychological measurement, 24(2), 239-253.

Hederich, Christian. (2004). Estilo cognitivo en la dimensión de dependencia-independencia de campo. Influencias culturales e implicaciones para la educación. (Tesis doctoral) Universidad Autónoma de Barcelona, Barcelona, España.

Hederich, Christian. (2010). Acerca de la noción general de estilo en la educaciónPertinencia, importancia y especificidad. Actualidades Pedagógicas, (55), 13-21.

Hermann, Ned. (1996). The whole brain business book. New York: McGraw-Hill. 
Hofstein, Avi, Ben-Zvi, Ruth \& Samuel, David. (1978). A Comparative Study of Cognitive Preferences of Different Groups of Chemistry Students. Journal of Chemical Education, 55 (11), 705-707.

Holzman, Philip \& Klein, George. (1954). Cognitive system-principles of leveling and sharpening: Individual differences in assimilation effects in visual time-error. Journal of Psychology Interdisciplinary and Applied, 37(1), 105-122.

Joyce, Bruce y Weill, Marsha. (1985). Modelos de Enseñanza. New Yersey, USA: Prentice Hall, Inc.

Kagan, Jerome. (1966). Reflection-impulsivity: The generality and dynamics of conceptual tempo. Journal of Abnormal Psychology, 71(1), 17-24.

Keller, Jean \& Ripoll, Hubert. (2001). Reflective-impulsive style and conceptual tempo in a gross-motor task. Perceptual and Motor Skills, 92(3), 739-749.

Kempa, Richard \& Dubé, G.E. (1973). Cognitive Preference Orientation in Students of Chemistry. The British Journal of Educational Psychology, 43(3), 279-288.

Kholodnaya, Marina. (2002). Kognitiivnii stili: O prirode individual'nogo uma [Cognitive styles: On the nature of individual mind]. Moscow: PER SE.

Kirschner, Paul. (1992). Epistemology, practical work and academic skills in science education. Science Education, 1, 273-299.

Klein, George. (1951). A personal world through perception. In Robert R. Blake and Glenn V. Ramsey (Eds.), Perception: An approach to personality (pp. 328-355). New York: The Ronald Press Company.

Klein, George \& Schlesinger, Herbert. (1951). Perceptual attitudes toward instability: I. Prediction of apparent movement experiences from Rorschach responses. Journal of Personality, 19(3), 289-302.

Kolb, Alice \& Kolb, David. (2005). The Kolb learning style inventory-version 3.12005 technical specifications. Boston, MA: Hay Group Transforming Learning.

Kolb, David. (2005). The Kolb learning style inventory-version 3.1: Self-scoring and interpretation booklet. Boston, MA: Hay Group Transforming Learning.

Kolb, David, Boyatzis, Richard \& Mainemelis, Charalampos. (2001). Experiential learning theory. Previous research and new directions. In R. J. Sternberg \& L. Zhang (Eds.), Perspectives on thinking, learning, and cognitive style: The educational psychology series (pp. 227-247). Mahwah, NJ: Erlbaum.

Kozhevnikov, María. (2007). Cognitive Styles in the Context of Modern Psychology: toward an integrated framework of cognitive style. Psychological bulletin, 133(3), 464-481.

Lamb, Steven \& Certo, Samuel. (1978) The Learning Style Inventory (LSI) and instrument bias. Academy of Management. Proceedings, 1, 28-32. 
Martínez, Pedro. (2009). Estilos de enseñanza: conceptualización e investigación (En función de los estilos de aprendizaje de Alonso, gallego y Honey). Estilos de Aprendizaje, 3(3), 3-19.

McRobbie, Campbell. (1991). Cognitive Styles and Cognitive Structure. Science Education, 75(2), 231-242.

Messick, Samuel. (1976). Personality consistencies in cognition and creativity. In Samuel Messick (Ed.), Individuality in learning: Implications of cognitive styles and creativity for human development (pp. 4-23). San Francisco: Jossey-Bass.

Moreira, Marco y Levandowski, Carlos. (1983). Diferentes abordagens ao ensino de laboratorio. Porto Alegre: Editora da Universidade.

Niaz, Mansoor. (1987). Mobility-fixity dimension in Witkin's theory of field dependenceindependence and its implication for problems solving in science. Perceptual and Motor Skills, 65(3), 755-764.

Nosal, Czeslaw. (1990). Psychologiczne modele umyslu [Psychological models of mind]. Warsaw, Poland: PWN.

Rendón, María. (2010). Una descripción de los estilos de enseñanza en la Universidad de Antioquia. Revista Uni-Pluri/versidad, 10(2), 1-19.

Rendón, María. (2013). Hacia una conceptualización de los estilos de enseñanza. Revista Colombiana de Educación, (64), 175-195.

Riding, Richard. (2002). School Learning and Cognitive Style. London: David Fulton Publishers.

Riding, Richard \& Rayner Stephen. (2002). Cognitive styles and Learning Strategies. London: David Fulton Publishers.

Siegel, Laurence \& Siegel, Lila. (1965). Educational set: A determinant of acquisition. Journal of Educational Psychology, 56(1), 1-12.

Tamir, Pinchas. (1981). Validation of cognitive preferences. British Educational Research Journal, 7(1), 37-49.

Vásquez, Ana y Martínez, Isabel. (1996). La socialización en la escuela: una perspectiva etnográfica. Barcelona: Paidós.

Witkin, Herman. (1950). Individual differences in ease of perception of embedded figures. Journal of Personality, 19(1), 1-15.

Witkin, Herman \& Ash, Solomon. (1948). Studies in space orientation: IV. Further experiments on perception of the upright with displaced visual field. Journal of Experimental Psychology, 38(6), 762-782. 
Witkin, Herman, Lewis, Helen, Hertzman, Max, Machover, Karen, Bretnall, P., \& Wapner, Seymour. (1954). Personality through perception: An experimental and clinical study. New York: Harper \& Brothers.

Witkin, Herman Moore, Carol, Goodenough, Donald, \& Cox, Patricia. (1977). Field dependent and field independent cognitive styles and their educational implications. Review of Educational Research, 47(1), 1-64. 